\title{
Beyond the semiclassical description of Bloch oscillations
}

\author{
F. Domínguez-Adame \\ GISC, Departamento de Física de Materiales, Universidad Complutense, E-28040 \\ Madrid, Spain \\ E-mail: adame@fis.ucm.es
}

\begin{abstract}
.
Electrons moving in a tilted periodic potential perform a periodic motion, known as Bloch oscillation. Within a semiclassical description, the crystal momentum increases linearly with time until it reaches the boundary of the first Brillouin zone in reciprocal space. Then, it reenters the first Brillouin zone by the opposite edge. This periodic motion in reciprocal space is acompanied by an oscillation in real space. The angular frequency of the oscillations and their amplitude can be calculated within the semiclassical framework. Nevertheless, the semiclassical approach cannot explain the rich phenomenology of the Bloch oscillations, like the breathing of the electronic wave packet. We present a simple description of the Bloch oscillations of tightly bound electrons in biased lattices at a basic level and calculate exactly the wave function as a function of time.
\end{abstract}

PACS numbers: 73.23.-b, 72.30.+q, 78.67.Lt

European Journal of Physics 31, 639 (2010) 


\section{Introduction}

The high-frequency response of solids under electromagnetic fields is to a large extent determined by the dynamics of the electrons, especially in metals and semiconductors. Thus, one of the main goals in introductory courses of Solid State Physics is to understand the details of the electron dynamics in a periodic potential subjected to external fields. At a quantum level, the analysis is difficult even within the one-electron approximation. For this reason, most introductory textbooks (e.g. Ref. [1]) rely on the semiclassical approach, earlier introduced by Bloch and Zener in this context [2, 3].

The semiclassical equation of motion for the crystal momentum $\boldsymbol{k}$ predicts that electrons in periodic potentials subjected to an applied electric field may undergo coherent oscillations $[2,3]$. They perform a periodic motion, in real and in $\boldsymbol{k}$-space, known as Bloch oscillations (BOs) [1, 4]. From semiclassical arguments it can be shown that BOs are characterized by a time period $\tau_{B}=2 \pi \hbar / e \mathcal{E} a$ and an amplitude $A_{B}=W / 2 e \mathcal{E}$, where $-e$ is the electron charge, $\mathcal{E}$ is the magnitude of the applied electric field, $a$ denotes the spatial period of the potential, and $W$ stands for the band width in the unbiased lattice. BOs were observed for the first time as coherent oscillations of electronic wave-packets in semiconductor superlattices $[5,6]$. These oscillations are related to the wave dynamics of particles, and therefore they can be observed in almost any coherent motion of waves in tilted periodic potentials. Thus, they were later detected as a periodic motion of ensembles of ultracold atoms [7, 8] and Bose-Einstein condensates $[9,10]$ in tilted optical lattices. BOs have also their countpart in optics [11] and acoustics [12].

The aim of this work is to fill the gap between the semiclassical approach and the fully quantum mechanical description based on the Schrödinger equation in the three dimentional space. We consider a single electron within the tight-binding approximation moving in a one-dimensional (1D) lattice subjected to a uniform electric field. Thefore, we will be dealing with a discrete equation for the amplitude of the wave function at every atom instead of a differential equation for the wave function itself.

\section{Wannier-Stark ladder}

We consider the electron states in an infinite 1D lattice under a uniform electric $\mathcal{E}$ applied along the lattice. Within the one-band tight-binding approximation, the amplitude of the wave function at lattice sites satisfies the following equation [13]

$$
E \psi_{n}=e \mathcal{E} a n \psi_{n}-J \psi_{n+1}-J \psi_{n-1},
$$

where $J$ is the hopping parameter. The energy of the atomic orbitals is set to zero without loss of generality. To simplify the notation we introduce the dimensionless energy $\epsilon=E / J$ and electric field $F=e \mathcal{E} a / J$. Therefore, Eq. (1) can be rewritten as

$$
\epsilon \psi_{n}=F n \psi_{n}-\left(\psi_{n+1}+\psi_{n-1}\right) .
$$


Recalling the recurrence relation of the Bessel functions (A.1) we can write the solution in the infinite lattice as

$$
\psi_{n}^{(k)}=J_{n-k}(2 / F), \quad k=0, \pm 1, \pm 2, \ldots,
$$

where the superscript $(k)$ labels the eigenstate. According to (A.2) these eigenstates are already normalized. From Eqs. (3) and (A.1) we also find that $\epsilon_{k}=F k$ and the corresponding eigenenergy is

$$
E_{k}=e \mathcal{E} a k .
$$

Therefore, the energy levels are equally spaced, $e \mathcal{E} a$ being the energy difference between them. This energy spectrum is known as the Wannier-Stark ladder. The Wannier-Stark ladder is also characterized by the spatial localization of the eigenstates. The degree of localization can be estimated from the second moment of the eigenstate $\psi_{n}^{(k)}$

$$
\begin{aligned}
(\Delta x)^{2} & =a^{2} \sum_{n} n^{2}\left|\psi_{n}\right|^{2}-a^{2}\left(\sum_{n} n\left|\psi_{n}\right|^{2}\right)^{2} \\
& =a^{2} S_{k}^{(2)}(2 / F)-a^{2}\left[S_{k}^{(1)}(2 / F)\right]^{2}=\frac{2 a^{2}}{F^{2}},
\end{aligned}
$$

where the sums $S_{k}^{(\ell)}(2 / F)=\sum_{n} n^{\ell} J_{n-k}^{2}(2 / F)$ are calculated in the Appendix. Thus, the width is finite and decreases on increasing the electric field as $\Delta x=\sqrt{2} \mathrm{~J} / \mathrm{e} \mathcal{E}$.

As an example, Fig. 1 shows the ingenfunction amplitude (3) as a function of the site index when $k=0$ and two values of the dimensionless electric field, namely $F=0.1$ and $F=1.0$. It becomes apparent that the spatial extent of the eigenstate is smaller on increasing the magnitude of the electric field. It is important to stress that other eigenstates are obtained by integer translations over the 1D lattice of those shown in Fig. 1 while their energies are quantized according to (4).

\section{Bloch oscillations}

Once the eigenstates have been obtained, we now focus on the time-dependent tightbinding equations

$$
i \hbar \frac{d \psi_{n}}{d t}=e \mathcal{E} a n \psi_{n}-J \psi_{n+1}-J \psi_{n-1}
$$

As in the previous section, we introduce the dimensionless electric field $F=e \mathcal{E} a / J$ as well as the dimensionless time $\tau=(J / \hbar) t$. We then rewrite (6) as follows

$$
i \dot{\psi}_{n}=F n \psi_{n}-\left(\psi_{n+1}+\psi_{n-1}\right)
$$

where the dot indicates the derivative with respect to $\tau$. To obtain the wave function we introduce the following combination of eigenstates

$$
\psi_{n}(t)=\sum_{k} c_{k} e^{i k F \tau} J_{n-k}(2 / F)
$$

Using the recurrence relation of the Bessel functions (A.1), it is a matter of simple algebra to prove that (8) is the solution of (7). 
Figure 1. Eingenfunction amplitude as a function of the site index when $k=0$ and a) $F=0.1$ and b) $F=1.0$. Solid lines are a guide to the eye.

The coefficients $c_{k}$ can be obtained from the initial wave packet $\psi_{n}(0)$ as follows. Multiplying (8) at $\tau=0$ by $J_{n-q}(2 / F)$ and performing a sum over all lattices sites $n$ we get

$$
c_{q}=\sum_{n} \psi_{n}(0) J_{n-q}(2 / F)
$$

Notice that the normalization of the wave packet is ensured if $\sum_{k}\left|c_{k}\right|^{2}=1$.

We now calculate the expected value of the electron position at time $t$, defined as follows

$$
x(t)=a \sum_{n} n\left|\psi_{n}(t)\right|^{2}=a \sum_{k} \sum_{q} c_{k} c_{q}^{*} \mathcal{S}_{k q} e^{i(k-q) F \tau}
$$

where $\mathcal{S}_{k q}$ is calculated in the Appendix [see Eq. (A.5)]. Therefore

$$
x(t)=a \sum_{k} k\left|c_{k}\right|^{2}+\frac{2 a}{F} \operatorname{Re}\left(e^{-i F \tau} \sum_{k} c_{k} c_{k+1}^{*}\right) .
$$

Finally, the centroid position, defined as $\xi(t)=x(t)-x(0)$, is obtained as follows

$$
\xi(t)=\frac{W}{2 e \mathcal{E}} \operatorname{Re}\left[\left(e^{-i \omega_{B} t}-1\right) \sum_{k} c_{k} c_{k+1}^{*}\right],
$$

where $\omega_{B}=e \mathcal{E} a / \hbar=2 \pi / \tau_{B}$ is the so called Bloch frequency and $W=4 J$ is the band width.

As working examples, we now consider three different initial conditions. 


\subsection{Electron initially localized at a single site}

In this case $\psi_{n}(0)=\delta_{n 0}$, where it is assumed that the electron is initially localized at $n=0$ without loss of generality. From (9) one gets $c_{k}=J_{-k}(2 / F)$ and the orthonormality condition (A.2) leads to $\xi(t)=0$. As a consequence, the expected position of the electron is the initial site at all times. The electron does not perform BOs, in the sense that the centroid remains located at the initial site. Nevertheless, the width of the wave packet varies in time. The calculation is lengthly but straightforward, and here we quote only the final result

$$
\Delta x(t)=\frac{1}{\sqrt{2}} \frac{W}{e \mathcal{E}}\left|\sin \left(\frac{\omega_{B} t}{2}\right)\right| .
$$

Therefore, the wave packet remains at rest but develops a breathing mode when the electron is initially localized at a single site.

\subsection{Two Wannier-Stark eigenstates}

Consider the situation when $c_{0}=c_{1}=1 / \sqrt{2}$ so the wave packet (8) is a superposition of two Wannier-Stark eigenstates

$$
\psi_{n}(t)=\frac{1}{\sqrt{2}}\left[J_{n}(2 / F)+e^{i \omega_{B} t} J_{n-1}(2 / F)\right] .
$$

The centroid position can be obtained from (12)

$$
\xi(t)=\frac{W}{4 e \mathcal{E}}\left(\cos \omega_{B} t-1\right)
$$

In constrast to the previous case, now the centroid oscillates harmonically with the Bloch frequency and the amplitude is given by $W / 4 e \mathcal{E}$. Figure 2 shows the squared wave function $\left|\psi_{n}(t)\right|^{2}$ as a function of the site index and time when $F=0.5$, where BOs in real space are clearly observed.

\subsection{Exponential wave packet}

As a third illustrative example we consider a wave packet that initially has an exponential profile of the form

$$
\psi_{n}(0)=\mathcal{N} e^{-|n| / \lambda}
$$

where the normalization constant is given by $\mathcal{N}^{-2}=\sum_{n} \exp (-2|n| / \lambda)$. From (9) and (12), the centroid position can be rewritten as

$$
\xi(t)=\frac{W}{2 e \mathcal{E}} \operatorname{Re}\left[\left(e^{-i \omega_{B} t}-1\right) \sum_{n} \psi_{n}(0) \psi_{n+1}^{*}(0)\right] .
$$

In the case of the exponential wave packet $\sum_{n} \psi_{n}(0) \psi_{n+1}^{*}(0)=\exp (-1 / \lambda)$. Therefore, the centroid is

$$
\xi(t)=\frac{W}{2 e \mathcal{E}} e^{-1 / \lambda}\left(\cos \omega_{B} t-1\right)
$$

As a conclusion, we observe that the exponential wave packet also performs BOs. 
Figure 2. Squared wave function $\left|\psi_{n}(t)\right|^{2}$ as a function of the site index and time (in units of the Bloch period, $\left.\tau_{B}\right)$ when $c_{0}=c_{1}=1 / \sqrt{2}$ and $F=0.5$.

It worth considering two limiting cases. In the first place, when the initial wave packet is strongly localized $(\lambda \rightarrow 0)$ the amplitude of the centroid motion vanishes. This result is in perfect agreement to that obtained in Sec. 3.1 when the initial wave packet was localized at a single site. In the opposite limit, when $\lambda \gg 1$, the exponential $e^{-1 / \lambda}$ can be safely replaced by unity and the amplitude is $W / 2 e \mathcal{E}$. In this case we recover the amplitude $A_{B}$ of the BOs obtained by semiclassical arguments (see the Introduction).

\section{Conclusions}

In conclusion, in this paper we have presented a detailed analysis of the dynamics of tightly bound electrons in a 1D lattice under an applied electric field. The equation for the wave function amplitude at lattice sites can be solved in the time-domain with the aid of the Wannier-Stark eigenstates. The semiclassical approach predicts that the electron performs BOs with well defined angular frequency and amplitude. However, our analysis has shown that the details of the dynamics depend on the initial conditions and, in some cases, are not well described by the semiclassical approach. For instance, the centroid of the wave packet does not oscillate at all when the electron is initially localized at a single site. The analysis requires a basic knowledge of special functions only. Therefore, we claim that it is suitable for introductory courses in Solid State Physics.

The authors thanks E. Díaz and C. González-Santander for helpful discussions. 
This work has been supported by MEC (project MOSAICO).

\section{Appendix A. Basic properties of Bessel functions}

Bessel functions satisfy the recurrence relation

$$
\frac{2 n}{x} J_{n}(x)=J_{n+1}(x)+J_{n-1}(x),
$$

and the orthonormality condition

$$
\sum_{n} J_{n-k}(x) J_{n-k^{\prime}}(x)=\delta_{k k^{\prime}} .
$$

In particular notice that $\sum_{n} J_{n}^{2}(x)=1$.

Bessel functions of positive and negative index are related by $J_{-n}(x)=(-1)^{n} J_{n}(x)$. Therefore

$$
S_{k}^{(1)}(x) \equiv \sum_{n} n J_{n-k}^{2}(x)=k \sum_{n} J_{n}^{2}(x)+\sum_{n} n J_{n}^{2}(x)=k .
$$

The last sum vanishes since $J_{-n}^{2}(x)=J_{n}^{2}(x)$ and positive and negative terms cancel each other. The width of the Wannier-Stark eigenfunctions is related to the following sum

$$
\begin{aligned}
S_{k}^{(2)}(x) & \equiv \sum_{n} n^{2} J_{n-k}^{2}(x)=\sum_{n} n^{2} J_{n}^{2}(x)+2 k S_{0}^{(1)}(x)+k^{2} \\
& =\left(\frac{x}{2}\right)^{2} \sum_{n}\left[J_{n+1}(x)+J_{n-1}(x)\right]^{2}+k^{2}=\frac{x^{2}}{2}+k^{2},
\end{aligned}
$$

where the recurrence relation (A.1) and the orthonormality condition (A.2) have been taken into account.

Finally, we calculate the following sum invoking again the recurrence relation (A.1) and the orthonormality condition (A.2)

$$
\begin{aligned}
\mathcal{S}_{k q} & =\sum_{n} n J_{n-k}(x) J_{n-q}(x)=k \delta_{k q}+\sum_{n} n J_{n}(x) J_{n-q+k}(x) \\
& =k \delta_{k q}+\frac{x}{2}\left[\sum_{n} J_{n+1}(x) J_{n-q+k}(x)+J_{n-1}(x) J_{n-q+k}(x)\right] \\
& =k \delta_{k q}+\frac{x}{2}\left(\delta_{k, q+1}+\delta_{k, q-1}\right) .
\end{aligned}
$$

\section{References}

[1] N. W. Ashcroft and N. D. Mermin, Solid State Physics (Saunders Colledge Publishers, New York, 1976), p. 213.

[2] F. Bloch, Z. Phys. 52, 555 (1928).

[3] C. Zener, Proc. R. Soc. London, Ser. A 145, 523 (1934).

[4] T. Hartmann, F. Keck, H. J. Korsch and S. Mossmann, New J. Phys. 6, 2 (2004).

[5] J. Feldmann, K. Leo, J. Shah, D. A. B. Miller, J. E. Cunningham, T. Meier, G. von Plessen, A. Schulze, P. Thomas and S. Schmitt-Rink, Phys. Rev. B 46, R7252 (1992).

[6] K. Leo, P. Haring Bolivar, F. Brüggemann, R. Schwedler and K. Köhler, Solid State Commun. 84, 943 (1992).

[7] M. BenDahan, E. Peik, J. Reichel, Y. Castin and C. Salomon, Phys. Rev. Lett. 76, 4508 (1996). 
[8] S. R. Wilkinson, C. F. Bharucha, K. W. Madison, Q. Niu and M. G. Raizen, Phys. Rev. Lett. 76, $4512(1996)$.

[9] B. P. Anderson and M. A. Kasevich, Science 282, 1686 (1998).

[10] Ch. Gaul, R. P. A. Lima, E. Díaz, C. A. Müller and F. Domínguez-Adame, Phys. Rev. Lett. 102, 255303 (2009).

[11] R. Sapienza, P. Costantino, D. S. Wiersma, M. Ghulinyan, C. J. Oton and L. Pavesi, Phys. Rev. Lett. 91, 263902 (2003).

[12] H. Sanchis-Alepuz, Y. A. Kosevich and J. Sánchez-Dehesa, Phys. Rev. Lett. 98, 134301 (2007).

[13] D. H. Dunlap and V. M. Kenkre, Phys. Lett. A 127, 438 (1988). 